\title{
Electrical Contacts to Individual Colloidal Semiconductor Nanorods
}

\author{
Paul-Emile Trudeau, Matt Sheldon, Virginia Altoe, A. Paul Alivisatos*
}

Department of Chemistry, University of California, Berkeley, and Materials Science

Division, Lawrence Berkeley National Laboratory, Berkeley, California 94720

alivis@berkeley.edu

\begin{abstract}
We report the results of charge transport studies on single CdTe nanocrystals contacted via evaporated Pd electrodes. Device charging energy, $\mathrm{E}_{\mathrm{c}}$, monitored as a function of electrode separation drops suddenly at separations below $\sim 55$ nm. This drop can be explained by chemical changes induced by the metal electrodes. This explanation is corroborated by ensemble X-Ray photoelectron spectroscopy (XPS) studies of CdTe films as well as single particle measurements by transmission electron microscopy (TEM) and energy dispersive X-Rays (EDX). Similar to robust optical behavior obtained when Nanocrystals are coated with a protective shell, we find that a protective $\mathrm{SiO}_{2}$ layer deposited between the nanocrystal and the electrode prevents interface reactions and an associated drop in $\mathrm{E}_{\mathrm{c}, \max }$. This observation of interface reactivity and its effect on electrical properties has important implications for the
\end{abstract}


integration of nanocrystals into conventional fabrication techniques and may enable novel nano-materials. 
Three-terminal electrical measurements can carefully probe the electronic structure of a wide range of mesoscopic systems and nanostructures. ${ }^{1,2}$ This stems from the ability to systematically adjust the energy required to add or remove a charge from a nanoscale object. At low temperatures and in a magnetic field one can also control the quantum or spin level that is being probed. ${ }^{1}$ Information obtained in this way provides perhaps the most detailed look into the effects of quantum confinement and dimensional control of semiconductor systems. When we investigate nanostructures in three terminal geometries, however, questions arise regarding the nature of the electrical contacts. Indeed as we study smaller and smaller nanostructures it becomes increasingly likely that the electrical contacts substantially modify the quantum object under study.

Our interest is in the electrical study of colloidal semiconductor nanocrystals. Due to their size-dependent optical properties and the ability to introduce them into diverse chemical and biological environments, colloidal dots and rods are one of the most important examples of controlled quantum structure available. ${ }^{3,4}$ This control comes at a price; the solution based preparation as well as complicated surface ligand chemistry makes reliable electrical contacts and reproducible measurements of these structures difficult.

Prior studies of CdSe nanocrystals and nanorods illustrate these difficulties. The nanostructures resist a unified description of quantum confinement effects by both optical and electrical characterization except by the most gentle experimental techniques. For example, scanning tunneling microscopy ${ }^{5}$ as well as break junction experiments ${ }^{6}$ show a well defined band gap, and are even capable of resolving level structure in sufficiently small nanocrystals. These results can be consistent with energy gaps and level structure 
observed with optical spectroscopies. ${ }^{5,7}$ However, individual nanocrystal electrical behavior is sensitive to local charge environment, ${ }^{8,9}$ and two terminal measurements must be interpreted with care if comparison to an absolute energy scale is desired. It is more challenging to reconcile the complex charging energy patterns obtained when nanocrystals are instead addressed by lithographically deposited electrodes with the band gaps and level structures obtained by these other methods. ${ }^{10}$ To explore the mechanism behind these differences, we present a systematic study of the electrical properties of single semiconductor nanocrystals addressed by lithographically defined electrodes in a three-terminal geometry. Specifically we track variations in nanorod charging energy as a function of electrode spacing for both bare and insulated nanocrystals.

We prepared CdTe nanocrystals as reported previously ${ }^{11}$ and deposited them from toluene solutions onto test chips. Nanocrystals were located with respect to predefined alignment markers and we used electron beam lithography to create source and drain contacts (5 nm Cr/ $45 \mathrm{~nm} \mathrm{Pd} \mathrm{).} \mathrm{An} \mathrm{Au} \mathrm{film} \mathrm{separated} \mathrm{from} \mathrm{the} \mathrm{device} \mathrm{by} 10 \mathrm{~nm}$ of $\mathrm{SiN}$ served as a back gate. A schematic of a single nanocrystal device is shown in Figure 1a. Electrode separation varied from 30 to $100 \mathrm{~nm}$ as measured by scanning electron microscopy (SEM) (Figure 1b). All results reported here were measured at $5 \mathrm{~K}$ in a Janis (STVP-100) He4 flow cryostat.

We measured source-drain current as a function of source-drain voltage, as well as gate voltage. To visualize the results, we plotted the differential conductance $\left(\mathrm{dI}_{\mathrm{sd}} / \mathrm{dV}_{\mathrm{sd}}\right)$ as a function of both $\mathrm{V}_{\mathrm{sd}}$ and $\mathrm{V}_{\mathrm{g}}$ (Fig 1c). This highlights regions of zero conductance, which represent stable single electron charge states on the nanocrystal. ${ }^{2}$ The energy to charge an 
object goes as $\mathrm{e}^{2} / 2 \mathrm{C}$, where $\mathrm{C}$ is capacitance and proportional to the size of the object. This confers an inverse relationship between the charging energy and the size of the region to be charged. Simply put, it is the energy required to offset the repulsive forces generated by adding an extra electron to the object. We determine this parameter experimentally. For each device, we measure $\mathrm{E}_{\mathrm{c}, \max }=\mathrm{eV}_{\mathrm{sd}, \max }$, where $\mathrm{V}_{\mathrm{sd} \text {,max }}$ is the voltage difference between the upper and lower limits of the largest region of zero conductance, outlined by the black line in Figure 1c.

The electrical behavior of the devices falls into two categories. In the first (Figure 1c) we observe evenly spaced, uniform charging energies. These regular coulomb diamonds suggest a single charged object with nearly continuous energy levels. In the second [supporting material], devices exhibit electrical behavior more consistent with multiple tunnel junctions in series viz. higher maximum charging energies, and a jagged profile along the diamond edges. ${ }^{2,12}$ For either case, we expect $\mathrm{E}_{\mathrm{c}, \max }$ to track well with the device dimensions.

We recorded $\mathrm{E}_{\mathrm{c}, \max }$ as a function of electrode separation, $\mathrm{L}_{\mathrm{t}}$, on the nanocrystal. In principle it is more accurate to track the volume of nanocrystal between the electrodes, however the SEM resolution limits the determination of significant differences in the nanocrystal diameter, which was $\sim 12 \mathrm{~nm}$ for all devices.

Figure 2 reveals that for electrode separations greater than $60 \mathrm{~nm}, \mathrm{E}_{\mathrm{c}, \max }$ increases as expected with decreasing electrode separation; however, below $\sim 55 \mathrm{~nm}$ the charging energy drops with decreasing electrode separation. This unexpected result can be accounted when we consider the possibility that there is a chemical transformation of the nanorod when the metal electrode is deposited on it, and that this zone of chemical 
transformation extends 20-30nm into the nanorod, as cartooned in Figures 2c-e. Several lines of evidence that support this picture are described below.

First, consider the electrical measurements themselves, in the context of the nanorod consisting of three zones. There are two "interaction zones" near each electrode where the nanorods are chemically modified, separated by a central zone that consists of pristine nanorod. The size of the outer interaction zones (red) remains roughly constant, limited presumably by a solid-state diffusion process. When electrode spacing $\left(\mathrm{L}_{t}\right)$ decreases, these interaction zones encroach on the center zone (orange). The energy to charge the center zone dominates $\mathrm{E}_{\mathrm{c}, \max }$ when its length is reduced below that of the outer zones. At small enough $\mathrm{L}_{\mathrm{t}}$, the chemically modified zones merge to span the entire $\mathrm{NC}$; the charging energy falls as the volume is now twice the size of a single interaction zone (Figure 2c). For $\mathrm{L}_{\mathrm{t}}<55 \mathrm{~nm}$ we observe mostly simple electrical behaviors. This corroborates our interpretation as it suggests multiple tunnel junctions in series $\left(L_{t}>55\right)$ give way to simple energy spectra of a singly charged zone for shorter electrode spacing, as in Figure 1c, $\left(\mathrm{L}_{\mathrm{t}}<55\right)$. The drop in $\mathrm{E}_{\mathrm{c}, \max }$ around $\sim 55 \mathrm{~nm}$ indicates that the interaction zones extend approximately $20-30 \mathrm{~nm}$ into the nanocrystal. We note that this interface is likely not abrupt, as cartooned in Figure 2, but a gradient whose sharpness and extent is defined by the chemistry of the electrode and the semiconductor.

The total energy to charge the device is approximately the sum of the individual charging energies of each region. For devices in series, stochastic level alignments lead to fluctuations in $\mathrm{E}_{\mathrm{c}, \max }$. Fractionally, these can be as high as $\mathrm{N}^{-1 / 2}$, where $\mathrm{N}$ is the number of zones. ${ }^{13}$ This contributes to the observed scatter in our $\mathrm{E}_{\mathrm{c}, \max }$ data. 
A second line of evidence that supports the above picture arises when we consider nanorods protected from reaction with the evaporated metal via a thin oxide barrier layer. We coated test chips of CdTe nanocrystals with a thin $5 \mathrm{~nm}$ layer of $\mathrm{SiO}_{2}$ before depositing the electrodes. This helped distinguish whether the discontinuity in Ecmax was due to a specific chemical interaction of $\mathrm{Pd}$ with $\mathrm{CdTe}$ at the interface, or rather an electrostatic effect of the device geometry. ${ }^{14}$ Figure $2 \mathrm{a}$ shows that for the protected nanorods, the charging energy simply increases with decreasing electrode separation, as expected. The trend in $\mathrm{E}_{\mathrm{c}, \text { max }}$ vs. $\mathrm{L}_{\mathrm{t}}$ is maintained for these $\mathrm{SiO}_{2}$-treated devices to the smallest $\mathrm{L}_{\mathrm{t}}$ achievable in our experiment, with no observed discontinuity. This shows that electrostatic effects and geometry are not responsible for the discontinuity in $\mathrm{E}_{\mathrm{c}, \max }$. The non-reactive $\mathrm{SiO}_{2}$ film arrests the compositional segmentation of the nanocrystal. The use of thermal deposition techniques to add the $\mathrm{SiO}_{2}$ layer admits the possibility of pinhole shorts. These allow relatively easy flow of charge carriers despite the thickness of the insulating layer, while still providing some barrier to atomic diffusion. Experiments are currently underway on more robustly protected nanocrystals.

The results and interpretation we present are consistent with prior studies describing the modification of the composition and electronic structure of bulk semiconductors in contact with metal electrodes. Differential scanning calorimetry has tracked the reactivity of CdTe surfaces with many metals. ${ }^{15}$ Consistent with thermodynamic arguments, these reactions occur at temperatures far lower than those required to thermally deposit metal electrodes during conventional lithography. Brillson ${ }^{16}$ and others $^{17,18}$ showed that bulk metal-CdTe interfaces react to form metal-Cd alloys or metalTe complexes. These reactions alter the local electronic structure and overall 
semiconductor device performance. Importantly, unfavored reactions can occur, but are limited to a $\sim 20 \mathrm{~nm}$ distance from the interface, ${ }^{16}$ a length scale similar to our findings. In fact, surface structure plays a key role in semiconductor reactivity. For example surface stoichiometry, controlled by etching ${ }^{19}$ tunes the reactivity of semiconductor surfaces. ${ }^{15}$ We note that we expect our nanocrystal surfaces to be quite reactive due to defects, dangling bonds and incomplete ligand coverage. We also note that the diameter of our nanorods is on the same order as the size of a bulk semiconductor surface.

To further confirm that the electrode reacts with the nanocrystal, we tracked changes in core electron binding energies of dense monolayer films of CdTe nanocrystals when Pd is evaporated on top, using X-Ray photoelectron spectroscopy (XPS). We considered films of nanocrystals with, and without a thin layer of Pd $(0.8 \mathrm{~nm})$. We compared these to films of nanocrystals protected by a $5 \mathrm{~nm} \mathrm{SiO}$ barrier both with, and without Pd. Figure 3 summarizes the results. When $\mathrm{Pd}$ is deposited directly on the nanocrystal film, we observe shifts to lower binding energy for $\mathrm{Cd} 3 \mathrm{~d}$ electrons as well as shifts to higher binding energy for Te $3 \mathrm{~d}$ electrons. This indicates a chemical change occurs in the nanocrystals only when they are in intimate contact with Pd. This further implies that $\mathrm{Cd}$ alloys with $\mathrm{Pd}$, in excellent agreement with previous results ${ }^{15,18}$. In the case of $\mathrm{SiO}_{2}$ protected nanocrystal films, we observe little to no shifts in $\mathrm{Cd}$ or Te binding energies. This highlights that shifts in binding energies originate from the interaction of the NC in direct contact with Pd.

Finally, we simultaneously spatially and chemically profiled the reaction zone between the metal electrode and individual nanorods. We cast films of CdTe nanocrystals onto $\mathrm{SiN}(30 \mathrm{~nm}$ ) TEM windows. Next, we deposited $50 \mathrm{~nm}$ of Pd in $100 \mathrm{~nm}$ wide strips. We 
investigated these samples using a transmission electron microscope (TEM) equipped to analyze energy dispersive x-rays (EDX). We measured line scans of elemental composition for single nanocrystals at the electrode-nanocrystal interface and along the length of the nanocrystal by tracking $\mathrm{K} \alpha$ emission from $\mathrm{Pd}, \mathrm{Cd}$ and $\mathrm{Te}$. The $\mathrm{K}$ lines were used instead of L to insure good separation between these elemental signals, and control samples indicate that we have good discrimination. Figure 4 highlights two representative cases. Figure 4a shows a TEM image (greyscale), line scan (red) and corresponding $\mathrm{Pd} \mathrm{K} \alpha$ (green) and $\mathrm{Cd} \mathrm{K} \alpha$ (blue) emissions. Pd is present throughout the nanocrystal. Consistent with our hypothesis, this indicates diffusion of Pd into the CdTe nanocrystal and concomitant alteration of the nanocrystal composition near the metalsemiconductor interface. Figure $4 \mathrm{~b}$ shows a representative control and highlights the good elemental discrimination, signal to noise, as well as excellent alignment between the intensity and elemental line scans.

Taken together the TEM, XPS and the differences between electrical data with and without $\mathrm{SiO}_{2}$ all point to a reaction between the electrode and the nanocrystal. Though this reactivity is general to all semiconductor surfaces - i.e. the first $\sim 10 \mathrm{~nm}-$ it has a dominant effect on the properties of nanocrystals. These results have several exciting implications for future work. First, since thin barrier layers prevent reactions with the electrodes, this opens a path towards three-terminal electrical measurements on quantum confined colloidal nanocrystals with diverse shapes, composition and connectivities. This should lead to further work that reconciles the differences between the optical and STM spectroscopies with the three-terminal electrical measurements, as well as more indepth studies of the electrical properties of the nanocrystals in general. Second, the 
chemical reactions between the electrodes and the nanorods may be exploited to deliberately create new types of very stable nanostructured electrical devices with interesting qualities in their own right.

Acknowledgement. We thank the UC Berkeley Microfabrication Lab for use of their facility. P.-E.T. thanks Canada's National Science and Engineering Research Council for a fellowship. M.S. thanks the Center of Integrated Nanomechanical Systems for a fellowship. This work was performed at the Molecular Foundry, Lawrence Berkeley National Laboratory, and was supported by the Office of Science, Office of Basic Energy Sciences of the U.S. Department of Energy under Contract No. DE-AC02--05CH11231.

Supporting Information Available. Additional stability diagrams. Additional EDX data. 

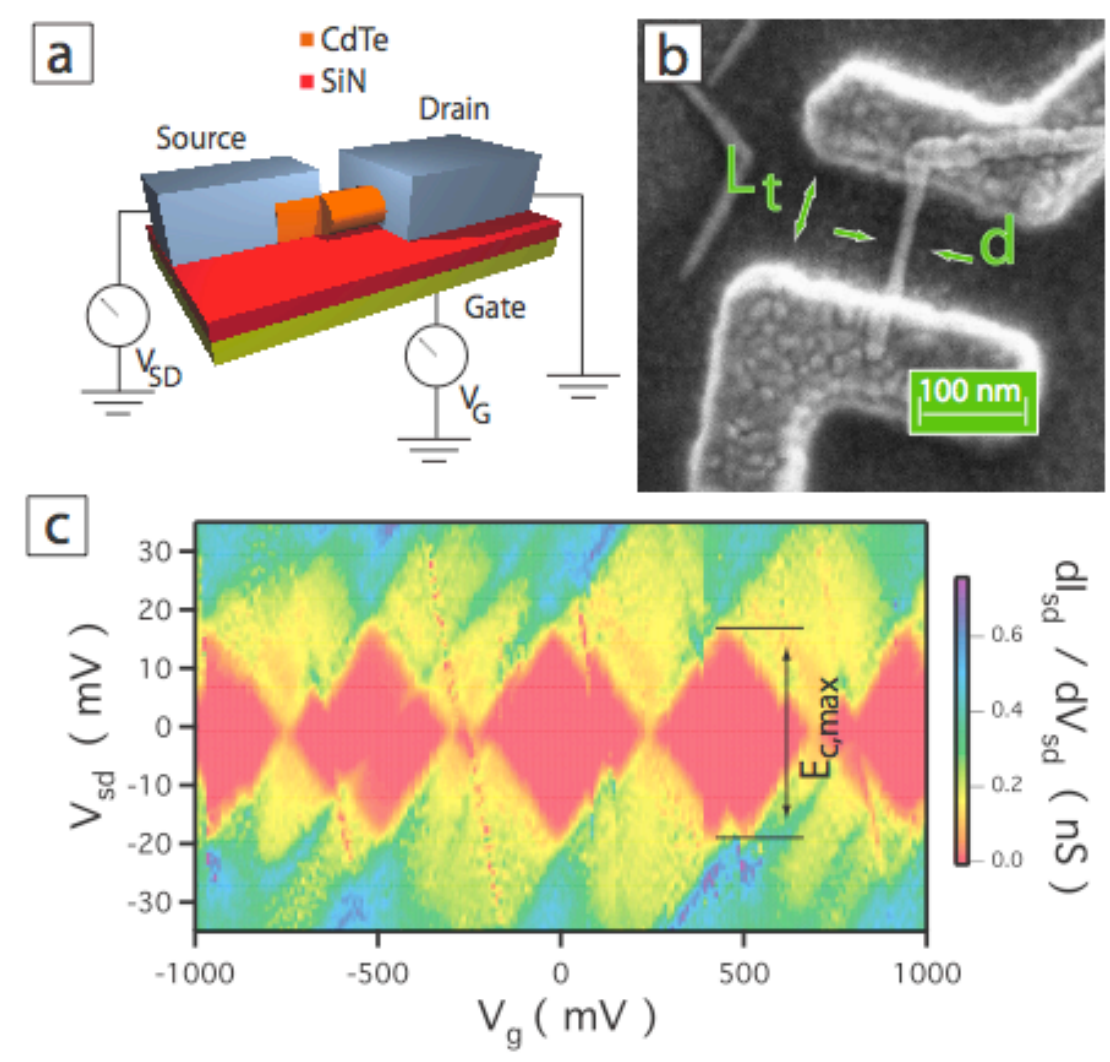

Figure 1. (a) Schematic of 3 terminal single nanocrystal (NC) device. The

Au gate is separated from the NC by $10 \mathrm{~nm}$ of SiN. Pd Source and drain electrodes are defined by e-beam lithography. (b) SEM image of single nanocrystal device of diameter, $d$, and total length, $\mathrm{L}_{\mathrm{t}}$. (c) Stability plot of single nanocrystal device. We extract the charging energy $\left(\mathrm{E}_{\mathrm{c} \max }\right)$ from the source-drain span of non-conducting (red) regions 


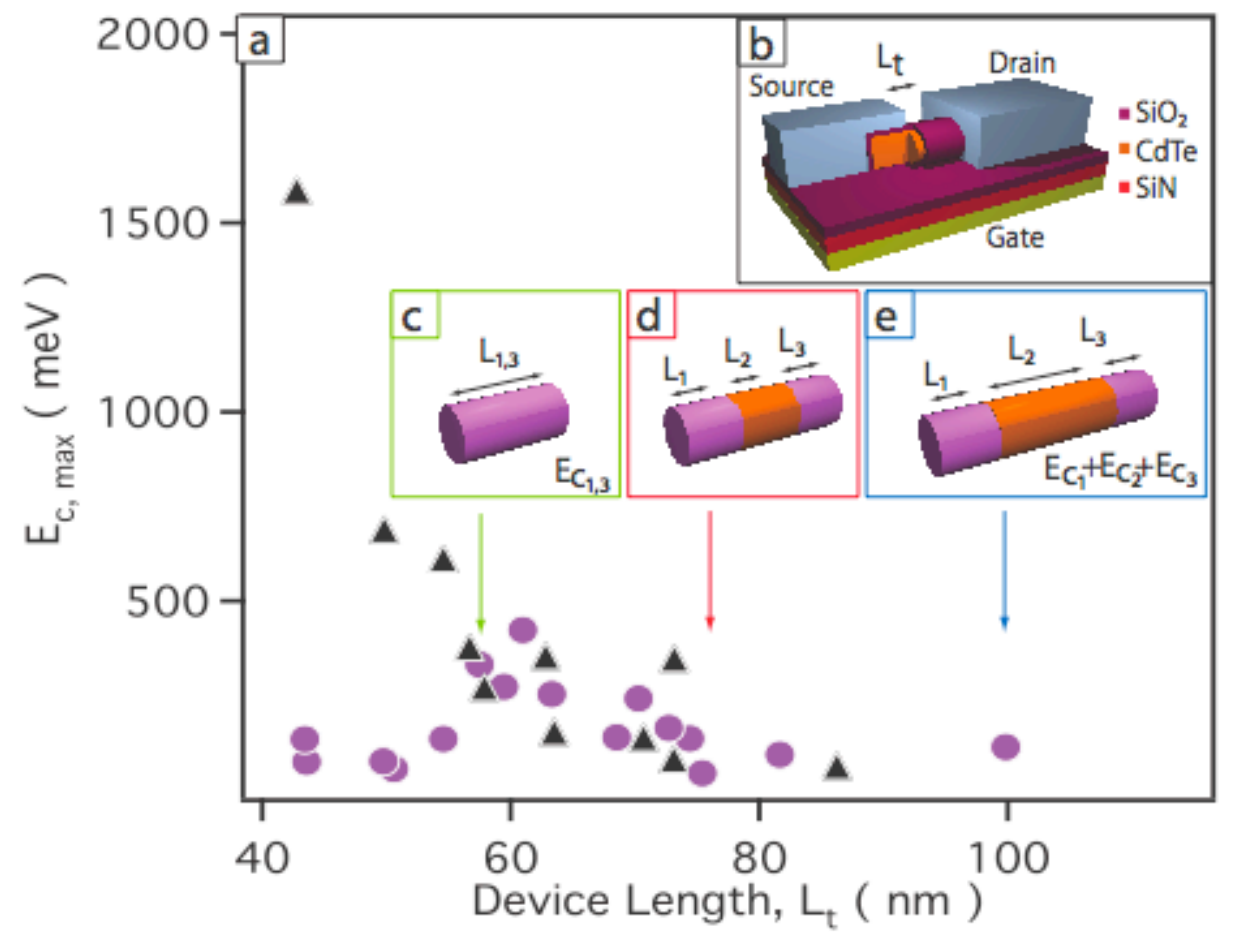

Figure 2. (a) Largest addition energy $\left(\mathrm{E}_{\mathrm{c}, \mathrm{max}}\right)$ of nanorod devices vs. electrode separation. Purple circles show devices not treated with $\mathrm{SiO}_{2}$. Black triangles show devices protected by $\mathrm{SiO}_{2}$ film as cartooned in (b). (c-e) Without $\mathrm{SiO}_{2}$ treatment, transport is determined by the interplay between zones of reacted $\mathrm{NC}$ near the electrodes (purple) and unreacted $\mathrm{NC}$ in the middle ( orange ). For sufficiently small $\mathrm{L}_{\mathrm{t}} \sim 55 \mathrm{~nm}$ (c) the reaction spans the NC and $\mathrm{E}_{\mathrm{c}, \max }$ drops. At larger $\mathrm{L}_{\mathrm{t}}(\mathrm{d}-\mathrm{e})$ charges pass from reacted to unreacted zones. This leads to 'in-series' contributions to the total addition energy $\left(\mathrm{E}_{\mathrm{c}, \max }\right)$. 


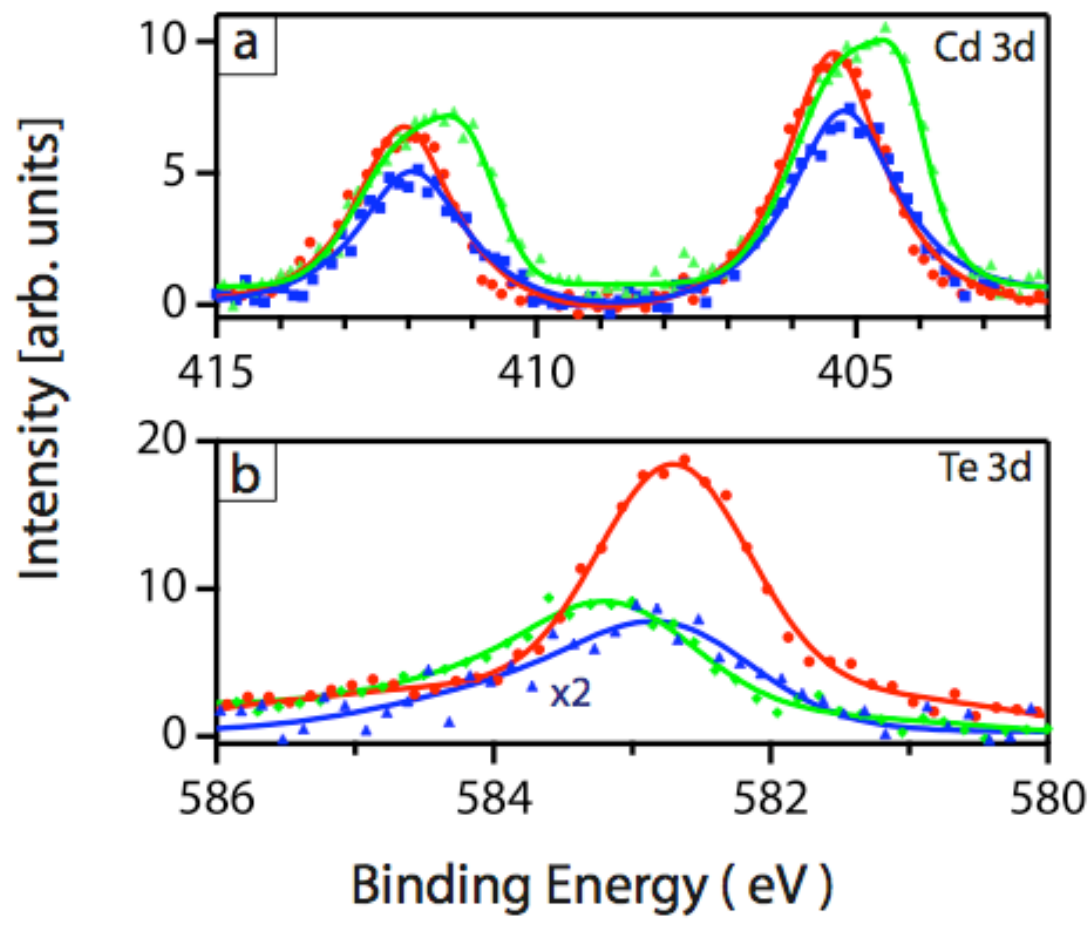

Figure 3. XPS data. Red curves are CdTe films, Blue curves are $\mathrm{SiO} 2$ coated CdTe films with $8 \AA$ Pd overlayer. Green curves are CdTe films with $8 \AA$ Pd overlayer. (a) Cd 3d emission reveals a shift to lower binding energies and Te $3 \mathrm{~d}$ emission. ( $\mathrm{b}$ ) shifts to higher energies only for unprotected ( green ) films. 


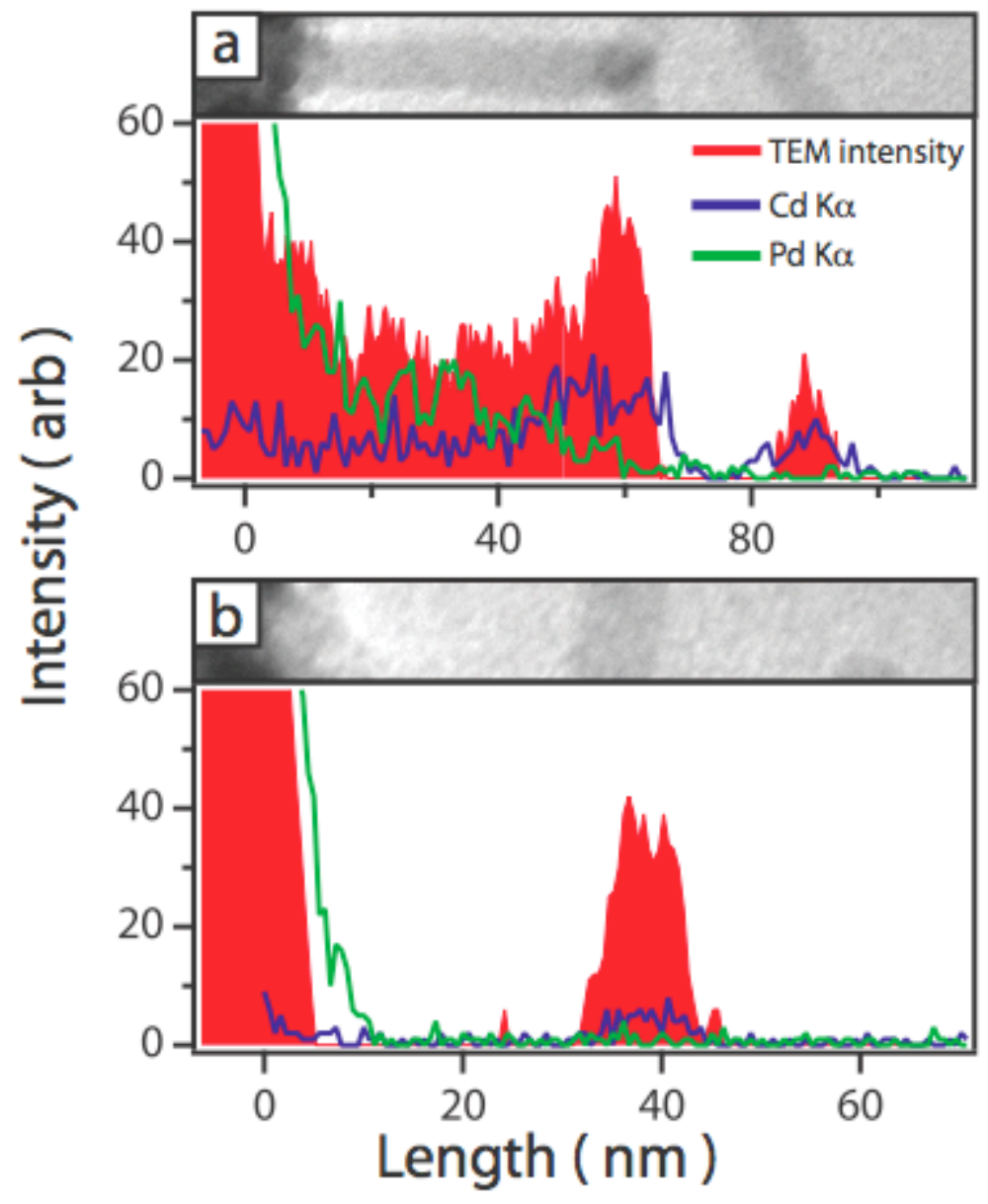

Figure 4. TEM data of CdTe NCs in contact with Pd. The intensity profile (taken from the center of the images) is show in red. $\mathrm{K} \alpha$ radiation from $\mathrm{Cd}$ (blue) and $\mathrm{Pd}$ (green) is superimposed. (a) Pd is present throughout much of the nanocrystal in contact with the electrode though not present at all in a NC not in contact with the electrode found at $\sim 80$ nm. (b) Control image shows excellent agreement between electrode edge as determined by both intensity and $\mathrm{Pd} \mathrm{K} \alpha$ signal. 


\section{REFERENCES}

(1) Single Charge Tunneling, Coulomb Blockade Phenomena in Nanostructures; Devoret, H. G. a. M. H., Ed.; Plenum Press: New York, 1992; Vol. 294.

(2) Averin, D. V.; Likharev, K. K. Journal of Low Temperature Physics 1986, 62,345 .

(3) Matsui, I. J. Chem. Eng. Japan 2005, 38, 535.

(4) Alivisatos, P. Nature Biotech 2004, 22, 47.

(5) Banin, U.; Millo, O. Annual Review of Physical Chemistry 2003, 54, 465.

(6) Klein, D. L.; Roth, R.; Lim, A. K. L.; Alivisatos, A. P.; McEuen, P. L. 1997, 389, 699.

(7) Nirmal, M.; Brus, L. Acc. Chem. Res. 1999, 32, 407.

(8) Hanna, A. E.; Tinkham, M. Physical Review B 1991, 44, 5919 LP

(9) Suganuma, Y.; Trudeau, P.-E.; Dhirani, A.-A. Physical Review B 2002, $66,241405$.

(10) Cui, Y.; Banin, U.; Bjork, M. T.; Alivisatos, A. P. Nano Lett. 2005, 5, 1519.

(11) Manna, L.; Milliron, D. J.; Meisel, A.; Scher, E. C.; Alivisatos, A. P. 2003, 2, 382. 
(12) van der Wiel, W. G.; De Franceschi, S.; Elzerman, J. M.; Fujisawa, T.; Tarucha, S.; Kouwenhoven, L. P. Reviews of Modern Physics 2002, 75, 1 LP

(13) Middleton, A. A.; Wingreen, N. S. Physical Review Letters 1993, 71, 3198

LP

(14) Demchenko, D. O.; Wang, L.-W. Nano Lett. 2007, 7, 3219.

(15) Lin, W.-Y.; Wei, C.; Rajeshwar, K. Journal of Applied Physics 1994, 76, 4145.

(16) Brillson, L. J. Physical Review Letters 1978, 40, 260 LP

(17) McGilp, J. F.; McGovern, I. T. Journal of Vacuum Science \& Technology B: Microelectronics and Nanometer Structures 1985, 3, 1641.

(18) Dharmadasa, I. M.; McLean, A. B.; Patterson, M. H.; Williams, R. H. Semiconductor Science and Technology 1987, 2, 404.

(19) Kotina, I. M.; Tukhkonen, L. M.; Patsekina, G. V.; Shchukarev, A. V.; Gusinskii, G. M. Semiconductor Science and Technology 1998, 13, 890.

(20) We believe that Pd is not on the NC surface. We discount small islands or even a thin surface skin, because this is not consistent with the observed low charging energies and simple electrical behavior at $\mathrm{L}_{\mathrm{t}}<55 \mathrm{~nm}$. 






\section{DISCLAIMER}

This document was prepared as an account of work sponsored by the United States Government. While this document is believed to contain correct information, neither the United States Government nor any agency thereof, nor The Regents of the University of California, nor any of their employees, makes any warranty, express or implied, or assumes any legal responsibility for the accuracy, completeness, or usefulness of any information, apparatus, product, or process disclosed, or represents that its use would not infringe privately owned rights. Reference herein to any specific commercial product, process, or service by its trade name, trademark, manufacturer, or otherwise, does not necessarily constitute or imply its endorsement, recommendation, or favoring by the United States Government or any agency thereof, or The Regents of the University of California. The views and opinions of authors expressed herein do not necessarily state or reflect those of the United States Government or any agency thereof or The Regents of the University of California. 